\title{
Low Work-Function Coating for an Entirely Propellantless Bare Electrodynamic Tether
}

\author{
John D. Williams, Juan R. Sanmartin, and Lauren P. Rand
}

\begin{abstract}
We present the possibility of a low work-function material, calcium aluminate electride, being used for a coating on a bare electrodynamic tether system. Analyses suggest that the coating would eliminate the need for an active cathodic device like a hollow cathode and, consequently, eliminate the need for an expellant to the hollow cathode, thus resulting in an electrodynamic tether system that requires no consumables. Applications include on-orbit power generation and deorbiting debris from low Earth orbit in a simple and trouble-free manner.
\end{abstract}

$\mathbf{t}$

\section{INTRODUCTION}

A $\mathrm{N}$ ELECTRON-EMITTER material, $12 \mathrm{CaO} \cdot 7 \mathrm{Al}_{2} \mathrm{O}_{3}$ (C12A7) electride, has been developed by Hosono's group at the University of Tokyo over the past decade (see [1]). Also called C12A7 electride, this crystalline inorganic electrically conducting ceramic is intriguing as a possible electron emitter because it has a very large lattice spacing, which may result in a material with a low work function.

Current state-of-the-art hollow cathodes use thermionic emission materials, such as $\mathrm{LaB}_{6}, \mathrm{CeB}_{6}$, and barium calcium aluminate impregnated porous tungsten (Ba-W) [2]. The work function of $\mathrm{LaB}_{6}$ and $\mathrm{CeB}_{6}$ is near $2.7 \mathrm{eV}$, while the work function of Ba-W is near $2.1 \mathrm{eV}$. In contrast, the effective work function of $\mathrm{C} 12 \mathrm{~A} 7$ electride has been reported to be as low as $0.6 \mathrm{eV}$ due to its unique nanocrystalline lattice structure [3]-[5]. $\mathrm{LaB}_{6}$ and $\mathrm{CeB}_{6}$ are generally heated to approximately $1900 \mathrm{~K}$ to obtain sufficient levels of emission, while $\mathrm{Ba}-\mathrm{W}$ is heated to $\sim 1300 \mathrm{~K}-1400 \mathrm{~K}$. These temperatures require expensive refractory heaters and good thermal insulation. In addition, the other cathode components must be made of materials capable of withstanding these temperatures. Ba-W cathodes, while operating at lower temperatures, are more susceptible to both poisoning and high rates of evaporation if operated at high current. Fig. 1 compares the plots

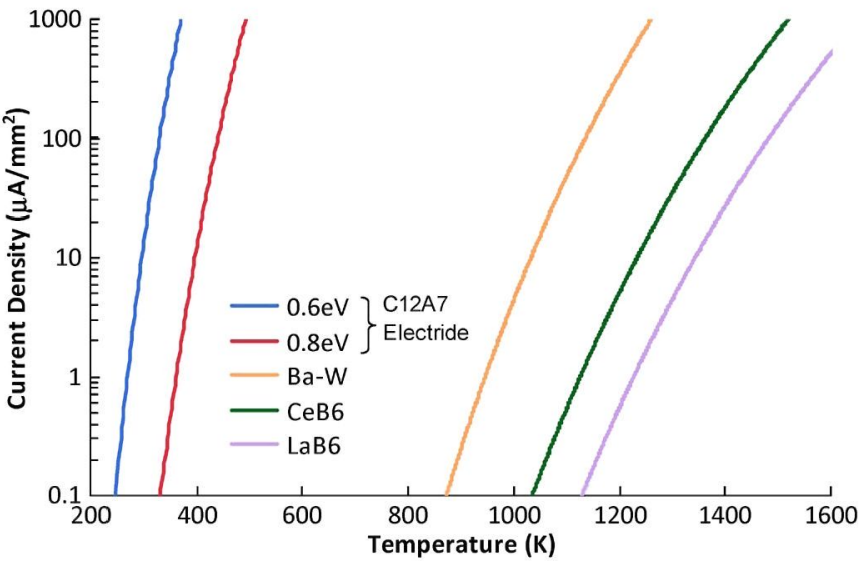

Fig. 1. Thermionic emission calculations of $0.6-$ and $0.8-\mathrm{eV}$ work-function materials compared with the emission calculations of commonly used thermionic materials in cathodes for intense electron-beam generation. Comparisons are made assuming Schottky-enhanced Richardson-Dushman emission (temperature-limited) at an electric field of $3.5 \mathrm{kV} / \mathrm{mm}$.

of various electron emitters operated in similar geometries and extraction voltages. The electride-emission curves at assumed work-function values of 0.6 and $0.8 \mathrm{eV}$ are shown to produce technologically interesting current-density levels at temperatures between $250 \mathrm{~K}$ and $500 \mathrm{~K}$. All electron emitters compared in Fig. 1 are assumed to be temperature-limited and to follow the Schottky-enhanced Richardson-Dushman emission law with the applied electric field set at $3.5 \mathrm{kV} / \mathrm{mm}$.

The promise of the low work function of $\mathrm{C} 12 \mathrm{~A} 7$ electride derives from its unique structure. The calcium aluminate phase C12A7 has been investigated for many decades. It is one of several phases found in common alumina-based cements. C12A7 has a naturally formed nanostructure, in which subnanometersized cages form a 3-D crystal lattice. The unit cell consists of 12 subcages. Although this cage structure is similar to those found in the clathrate phases of ice and in zeolites, there is a difference in that the unit cell of $\mathrm{C} 12 \mathrm{~A} 7$ is positively charged to $4+$. In other words, there are four fewer electrons on the atoms that comprise the framework cage of $\mathrm{C} 12 \mathrm{~A} 7$ than are needed to neutralize the cage. Interestingly, the positive charge in common $\mathrm{C} 12 \mathrm{~A} 7$ is counteracted by two atomic oxygen ions $\left(\mathrm{O}^{2-}\right)$ that are clathrated (floating) within two of the 12 subcages. New electrical and optical properties can be imparted to $\mathrm{C} 12 \mathrm{~A} 7$ if the free oxygen ions are substituted with anions like $\mathrm{O}^{-}$and $\mathrm{H}^{-}$, but particularly interesting features are possible when four electrons are substituted for the two $\mathrm{O}^{2-}$ ions to form $\mathrm{C} 12 \mathrm{~A} 7: \mathrm{e}^{-}$, the first inorganic electride discovered that is stable at high temperature [6], [7]. 


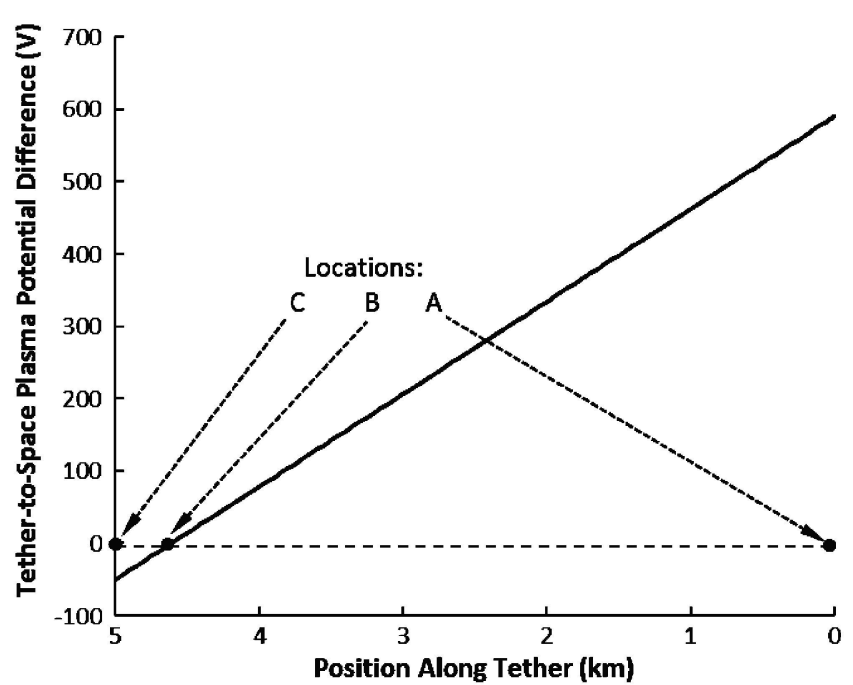

(a) Tether with active cathodic device or low work fuction coating.

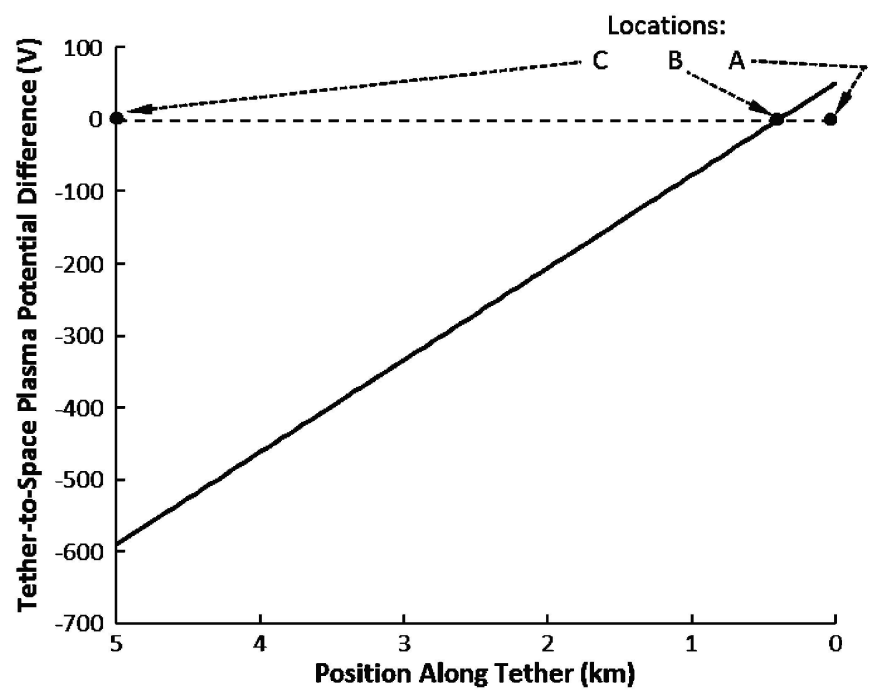

(b) Tether electrically floating

Fig. 2. Tether bias to space plasma situations for tethers (a) with electron emission and (b) without electron emission. Note that the values shown are arbitrary and do not correspond to a detailed analysis. (a) Tether with active cathodic device or low work-function coating. (b) Tether electrically floating.

\section{FloAting BARE TETHERS}

In a standard bare tether, one avoids using active devices for electron collection as the bare tether itself can collect electrons over a segment of its length that is positively biased of the space plasma. In the simplest drag mode, the positive (anodic) segment nearly covers the entire tether length, with a low-impedance active device at the cathodic end, such as a hollow cathode, designed to emit the full electron current that is collected on the positively biased segment of the tether. See Fig. 2(a) for a depiction of this tether configuration. In the absence of a low-impedance device, the tether is said to be passively electrically floating, and the current flowing along the tether vanishes at both ends. In contrast to a tether with an active cathodic emitter, the passively electrically floating condition is attained by ion collection from the space plasma over a long negatively biased segment, with a zero-bias point self-consistently located very close to the upper end of the tether as shown in Fig. 2(b).
In a completely passive electrically floating bare-tether system under OML current collection, the positive-bias length fraction is very small, about 0.03 (the cube root of electronto-ion mass ratio assuming atomic oxygen ions), because the ions are much heavier than the electrons. This results in the length-averaged current being much weaker than the current obtained if an active cathodic device is used and a long positively biased segment is created. Passive floating bare tethers have been proposed for creating artificial auroras and for the remote determination of the densities of neutral species in the ionospheric E-layer [8]. They are poor, however, at producing drag because of the low current level.

Thermionic emission by a floating bare tether might be more efficient than ion collection if a coating with low-enough work function $W$ is available and the tether temperature $T$ is high enough, for example, $0.6 \mathrm{eV}$ and $300 \mathrm{~K}$, respectively. In fact, at a very low work function, a condition may be attained that is opposite floating by ion collection, with a moderately short negative-bias length fraction resulting as if using an active cathodic device. Such a tether system could be said to be truly "propellantless," similar to field emitter array-based systems because no gas feed to a hollow cathode, for example, would be required.

There are two effects affecting, oppositely, thermal emission. The Schottky effect enhances emission by means of the electric field at the tether surface $E_{s}$ which, in principle, could be selfconsistently described by a double-layer sheath. The electric field $E_{s}$ would likely vary along the negative segment, and we judge that $E_{s}$ would be larger in magnitude as one moves toward the negatively biased end of the tether, which would enhance the Schottky effect. Separately, the space charge of the emitted electrons limits the emission current, with the Schottkyenhanced thermionic law only applying when its estimation of the electron-emission current is exceeded by the space-chargelimited current. As a location on the tether is biased more negatively, the space-charge-limited current will increase.

In this preliminary analysis, we will neglect ohmic effects for simplicity. In the tether frame, the tether is then equipotential, whereas the motional electric field $E_{m}$ holds in the ambient plasma. This results in a tether-to-plasma local bias $\Delta V$ decreasing linearly from the anodic end, $\mathrm{A}$, at $s=0$ to the cathodic end, C, at $s=L$ at the rate of that field $E_{m}$. The tetherto-ambient-plasma bias will vanish at some intermediate point, B, at $s=L_{B}$, which must be determined from the floatingtether condition.

\section{AnOdic-Segment Collection}

We assume that electron collection is given by the high-bias OML law, which will indeed hold, except at points very close to $\mathrm{B}$, for a round tether of radius $R$ less than a certain maximum value $R_{\max }$ that is close to the Debye length $\lambda_{D}$ for a typical ion-to-electron temperature ratio $T_{i} / T_{e} \approx 1$ [9]. The electroncollection current density in the $\mathrm{AB}$ segment, at distance $s$ from end $\mathrm{A}$, then reads

$j_{\mathrm{OML}}(s)=\frac{e N_{0}}{\pi} \sqrt{\frac{2 e \Delta V}{m_{e}}} \quad \Delta V=E_{m}\left(L_{B}-s\right)>0$ 
where $N_{0}$ is the unperturbed plasma density and where we assumed $e \Delta V \gg k T_{e} \sim 0.1 \mathrm{eV}$. The maximum of $j_{\mathrm{OML}}$ occurs at point $\mathrm{A}$

$$
j_{\mathrm{OML}}^{A}=\frac{e N_{0}}{\pi} \sqrt{\frac{2 e \Delta V_{A}}{m_{e}}}=\frac{e N_{0}}{\pi} \sqrt{\frac{2 e E_{m} L_{B}}{m_{e}}}
$$

where $L_{B}<L \equiv L_{B}+\Delta L$ is the length of segment $\mathrm{AB}$. At values typically considered in tether applications, $N_{0}=3 \times$ $10^{11} / \mathrm{m}^{3}, E_{m}=150 \mathrm{~V} / \mathrm{km}$, and $L_{B}=10 \mathrm{~km}$; the maximum current density for collection would be

$$
j_{\mathrm{OML}}^{A}=3.51 \times 10^{-7} \mathrm{~A} / \mathrm{mm}^{2} .
$$

From (1), one gets the rate of change of current $I(s)$ in a tether of cross-sectional perimeter $p$

$$
\frac{d I}{d s}=p \times \frac{e N_{0}}{\pi} \sqrt{\frac{2 e E_{m}\left(L_{B}-s\right)}{m_{e}}}
$$

yielding, after the integration and use of condition $I(0)=0$, the current at point $B$

$$
I_{B}=p \times \frac{e N_{0}}{\pi} \sqrt{\frac{2 e E_{m} L_{B}}{m_{e}}} \times \frac{2}{3} L_{B} \equiv p \times j_{\mathrm{OML}}^{A} \times \frac{2}{3} L_{B} .
$$

As the total collected electron current flows from point B into the negatively biased sections of the tether, it will be emitted and will gradually diminish to zero at point $\mathrm{C}$. The following sections describe this process.

\section{Cathodic-Segment Emission}

The thermionic current density reads

$$
j_{\mathrm{th}}=j_{0}(T, W) \times S, \quad S \equiv \exp \left[\frac{e}{k T} \sqrt{\frac{e E_{s}}{4 \pi \varepsilon_{0}}}\right]
$$

where $S$ is the Schottky enhancement factor and where the Richardson-Dushman current density $j_{0}$ is

$$
j_{0}=\frac{4 \pi m_{e} e k^{2}}{h^{3}} \times T^{2} \exp \left(-\frac{W}{k T}\right) .
$$

The approximate value of the universal first ratio in $j_{0}$ is $1.20 \mathrm{~A} / \mathrm{mm}^{2} \cdot \mathrm{K}^{2}$. We will here assume, for simplicity, that the Schottky effect is negligible (that is $S=1$ ), a condition that can be later checked out.

With the effects of thermal conduction in long tethers negligible, the tether temperature $T$ entering the Richardson-Dushman law results, in general, from a local balance between heating processes and radiation cooling. Heating over the positive segment is due to both the impact of electrons collected and the ohmic dissipation and, at daytime, solar irradiation. In the negative segment, impact heating may be ignored. For weak ohmic effects, as we are assuming, thermal emission might then be negligible at nighttime. For a tentative daytime temperature of $300 \mathrm{~K}$ and coating with $W$ as low as $0.6 \mathrm{eV}$, the RichardsonDushman current density would be

$$
j_{0}(300 \mathrm{~K}, 0.6 \mathrm{eV})=0.907 \times 10^{-5} \mathrm{~A} / \mathrm{mm}^{2} .
$$

The thermal-emission current density will be locally given by whichever is the lowest between the thermionic $j_{\text {th }}$ and the space-charge-limited $j_{\mathrm{SCL}}$ current densities. The space-chargelimited current-per-unit length, for emission from a uniformly biased inner cylinder of radius $r_{\text {cat }}$ to a nonemissive outer coaxial cylinder of radius $r_{\text {an }}$ [10], reads

$$
i=4 \pi \varepsilon_{0} \times \frac{2 \Phi(r)}{9 r\left[\beta\left(r_{\text {cat }} / r\right)\right]^{2}} \times \sqrt{\frac{2 e \Phi(r)}{m_{e}}}, \quad r_{\text {cat }}<r<r_{\text {an }}
$$

where initial velocities at emission have been ignored, $i$ is independent of $r$, and $\Phi(r)>0$ is the potential increase from $r_{\text {cat }}$ to $r$. The function $\beta$ vanishes along with $\Phi$ at $r_{\text {cat }}$, but as $r$ increases, $\beta$ increases, too. In general, one has $\Phi / \mathrm{r}^{2 / 3} \propto\left[\beta\left(r_{\text {cat }} / r\right)\right]^{4 / 3}$. For large-enough $r_{\text {an }} / r_{\text {cat }}, \beta^{2}$ is very close to one beyond, for example, $r / r_{\text {cat }}>10$, and we then have $\Phi \propto r^{2 / 3}$ [10]. Evaluating $i$ at $r_{\text {an }}$ and introducing the cathode perimeter $p=2 \pi r_{\text {cat }}$, the current density there, i.e., the space-charge-limited current density at emission, reads

$$
j_{\mathrm{SCL}}=\frac{4 \pi \varepsilon_{0}}{2 \pi r_{\mathrm{cat}}} \times \frac{2 \Phi_{\mathrm{an}}}{9 r_{\mathrm{an}}} \times \sqrt{\frac{2 e \Phi_{\mathrm{an}}}{m_{e}}} .
$$

\section{Cathodic-SEgment Double LAyer}

In some situations, tethers with a limited length or those operated in highly inclined orbits or at high altitude will not be able to collect very significant electron currents on the positively biased segment from A to $\mathrm{B}$. In these situations, it is possible that the entire length from $\mathrm{B}$ to $\mathrm{C}$ will be operating under electron-emission conditions that are limited by spacecharge effects. This situation is entitled "short cathodic segment," and its corresponding analysis is described hereinafter. In other tether geometry or orbital situations, the cathodic length will be longer and will include separate regions where space-charge-limited current flow will apply and where the temperature-limited current will apply. This situation is entitled "long cathodic segment," and its analysis is also described hereinafter.

The cathodic segment $\mathrm{BC}$ of the tether will exhibit a double layer, with ambient-plasma ions arriving at the tether and with the tether emitting electrons. While the small OML ion current is indeed negligible, we will go further in simplifying this preliminary analysis by using (10), which ignores the ion density in the sheath, although the sheath structure is used in making definite that very equation. We will use, as a rough model for $r_{\mathrm{an}}$, the sheath radius $r_{\mathrm{sh}}$ arising from the analysis of ion $O M L$ collection, which is [9]

$$
r_{\mathrm{sh}}=\frac{R}{\sqrt{\sigma_{1}\left(R / R_{\max }\right)}} \sqrt{\frac{e|\Delta V|}{k T_{e}}}
$$

where $\sigma_{1}$ is a monotonically increasing function of $R / R_{\max }$, which equals 0.24 for $R=R_{\max }$. (The tether radius is $R$, and $R_{\max }$ is the maximum radius for the OML regime to hold.) Setting $\Phi_{a, n}=|\Delta V|$ and $r_{\text {cat }}=R$, (10) yields a current density at emission that is linear in bias $\Delta V$ (except very 
$j_{\mathrm{SCL}}=\frac{4}{9} \sqrt{\sigma_{1}} \sqrt{\frac{2 k T_{e}}{m_{e}}} \frac{\varepsilon_{0}|\Delta V|}{R^{2}}, \quad|\Delta V|=E_{m}\left(s-L_{B}\right)>0$.

For some range beyond point $\mathrm{B}$, with $\Delta \mathrm{V}$ magnitude small and $R$ moderate, we will have space-charge-limited emission, with $j_{\mathrm{SCL}}<j_{0}$

$j_{\mathrm{SCL}}(s) \equiv j_{*} \frac{s-L_{B}}{L_{B}}, \quad$ with $j_{*} \equiv \frac{4}{9} \sqrt{\sigma_{1}} \sqrt{\frac{2 k T_{e}}{m_{e}}} \frac{\varepsilon_{0} E_{m} L_{B}}{R^{2}}$.

The integration of the rate of change of the current in the tether

$$
\frac{d I}{d s}=-p \times j_{\mathrm{SCL}}(s)
$$

leads to

$$
I(s)=I_{B}-p \frac{j_{*}\left(s-L_{B}\right)^{2}}{2 L_{B}} .
$$

Let a length $s-L_{B}=l$ be defined by the condition

$$
j_{\mathrm{SCL}}\left(L_{B}+l\right) \equiv j_{*} \times \frac{l}{L_{B}}=j_{0} .
$$

With (14) and (15) valid, where $j_{\mathrm{SCL}}<j_{o}$, the length $l$ defines the boundary with a jSCL $>j_{0}$ region.

\section{A. Short Cathodic Segment}

For length $\Delta L \equiv L-L_{B}$ smaller than $l$, (14) holds all the way to point $C$. The floating-tether condition now reads

$$
I_{C}=I(L)=I_{B}-\frac{p j_{*}}{2 L_{B}}|\Delta L|^{2}=0
$$

yielding, after use of (5)

$$
\frac{\Delta L}{L_{B}}=\frac{\sqrt{3 / \pi}}{\sigma_{1}^{1 / 4}} \times\left(\frac{k T_{e}}{e E_{m} L_{B}}\right)^{1 / 4} \frac{R}{\lambda_{D}} .
$$

For $R=\lambda_{D} \approx R_{\max }$, we have

$$
\Delta L / L_{B} \approx 1.4\left(k T_{e} / e E_{m} L_{B}\right)^{1 / 4}
$$

yielding a cathodic-segment length that is about $10 \%$ of the total length $L$ for a typical ratio $k T_{e} / e E_{m} L_{B}$ on the order of $10^{-4}$ and is weakly dependent on that ratio. This proves SCL emission to be indeed efficient. Note, further, that for a typical Debye length of several millimeters, practical (not too heavy) round wires would have $R / \lambda_{D}$ definitely less than one, making for a smaller $\Delta L / L_{B}$ ratio.

\section{B. Long Cathodic Segment}

For length $\Delta L \equiv L-L_{B}$ larger than $l,(14)$ breaks down at a point $\mathrm{B}^{*}$ between points $\mathrm{B}$ and $\mathrm{C}$, at distance $l$ from $\mathrm{B}$, with a

$$
I_{B^{*}}=I_{B}-\frac{p j_{*}}{2 L_{B}} l^{2}>0 .
$$

In the range $L_{B}+l<s<L_{B}+\Delta L$, we have

$\frac{d I}{d s}=-p \times j_{0}(T, W) \quad \Rightarrow \quad I(s)=I_{B^{*}}-p j_{0}\left[s-\left(L_{B}+l\right)\right]$.

The floating condition now reads

$$
I(L)=I_{B^{*}}-p j_{0}\left[L-\left(L_{B}+l\right)\right]=0
$$

or, using (16) and (20)

$$
I_{B}=p j_{0}\left(\Delta L-\frac{1}{2} l\right) .
$$

At $\Delta L=l,(17)$ and (23) give a common result, $I_{B}=$ $p L_{B} j_{0}^{2} / 2 j_{*}$.

The use of (5) in (23) yields $\Delta L / L_{B}$ for the long cathodicsegment regime. A joint law for both long and short regimes can be readily derived

$$
f\left(\frac{L_{B} j_{0}}{\Delta L j_{*}}\right) \times \frac{\Delta L}{L_{B}}=\frac{\sqrt{3 / \pi}}{\sigma_{1}^{1 / 4}} \times\left(\frac{k T_{e}}{e E_{m} L_{B}}\right)^{1 / 4} \frac{R}{\lambda_{D}}
$$

where

$$
\begin{array}{ll}
f(x)=1 \text { for } x>1 & \text { (short cathodic segment); } \\
f(x)=\sqrt{x(2-x)} \text { for } x<1 & \text { (long cathodic segment). }
\end{array}
$$

\section{Short/Long Cathodic-Segment Transition}

Equation (18a) shows how moving into the long cathodic regime increases the $\Delta L / L_{B}$ ratio. For $\Delta L=2 l(x=1 / 2)$ at a given right-hand side of (18a), that ratio increases by just $15 \%$. At the regime transition, i.e., setting $\Delta L=l$ in either (18) or (18a), we have

$$
\frac{\sqrt{3 / \pi}}{\sigma_{1}^{1 / 4}} \times\left(\frac{k T_{e}}{e E_{m} L_{B}}\right)^{1 / 4} \frac{R}{\lambda_{D}}=\frac{l}{L_{B}}=\frac{j_{0}}{j_{*}} .
$$

From (24), the full short cathodic-segment regime, corresponding to condition $\Delta L \leq l$, can be written as

$$
\sqrt{\frac{32}{27 \pi}} \sigma_{1}^{1 / 4} \times\left(\frac{k T_{e}}{e E_{m} L_{B}}\right)^{1 / 4} \frac{\omega_{p e}}{R} \varepsilon_{0} E_{m} L_{B} \leq j_{0}(T, W) .
$$

For $E_{M} L_{B}=1.5 \mathrm{kV}, N_{0}=3 \times 10^{11} / \mathrm{m}^{3}, T_{e}=0.1 \mathrm{eV}$, and $R=\lambda_{D}=4.29 \mathrm{~mm},(24 \mathrm{a})$ reads

$$
0.372 \times 10^{-5} \mathrm{~A} / \mathrm{mm}^{2} \leq j_{0}(T, W)
$$

which is satisfied by the thermionic current density given in (8).

A low value of the left-hand side of (24a) is indeed convenient because it would allow the use of materials with work function $W$ not as low as $0.6 \mathrm{eV}$. We also note that gross round tethers with a radius of $R=4.29 \mathrm{~mm}$ are impractical due to their excessive mass. On the other hand, a thin-tape tether could have that large a value for $R_{\text {eq }}$ (which is $1 / 4$ tape width) while collecting OML current proportional to the 
twice-width perimeter [9]. Note, however, that $\omega_{\mathrm{pe}} / R$ with $R=$ $\lambda_{D}$ is proportional to $N_{0}$. In moving to a larger day density, $N_{0}=10^{12} / \mathrm{m}^{3}$, the left-hand side of (24a) increases to a value $1.24 \times 10^{-5} \mathrm{~A} / \mathrm{mm}^{2}$, which does exceed $j_{0}$ in (8). Finally, a thin wire of radius $1 \mathrm{~mm}$ in a plasma of density $10^{12} / \mathrm{m}^{3}$ would definitely fall in the long cathodic-segment regime.

Fortunately, ohmic effects, ignored up to here in the analysis, appear to help in using electron thermal emission. A thin-tape tether at high plasma day densities may approach the shortcircuit current $\left(\sigma_{c} E_{M} w h\right.$, where $\sigma_{c}$ is the tether conductivity, $w$ is the tape width, and $h$ is the tape thickness) not far from A, the anodic end. In a typical tether system that uses a hollow cathode device with a very low impedance path back to the space plasma, the zero-bias point, $\mathrm{B}$, occurs very near $\mathrm{C}$, the cathodic end of the tether. This means that the tether potential is close to the ambient potential over most of the tether length and where negligible electron collection would occur. With thermal emission replacing hollow cathode use, point $\mathrm{B}$ would move closer to $\mathrm{A}$, the anodic end, with no reduction in maximum current within the tether and little reduction in length-averaged current. The detailed analysis of the ohmic-dominated case is left to future work.

\section{CONClusion}

A new material, calcium aluminate $\mathrm{C} 12 \mathrm{~A} 7$ electride, with a possible work function as low as $0.6 \mathrm{eV}$, has the potential to allow a completely floating bare-tether system to be developed that would display superior performance for deorbiting LEO satellite systems. Our analysis has shown that a coating of a low work-function material would result in a short cathodic section, high drag generation, and elimination of the need for an active cathodic device and its corresponding gas feed requirements. Operating at only $300 \mathrm{~K}$, the low work-function coating emits sufficient electrons to meet the demands of a typical flat-ribbon bare-tether system. In fact, most of the cathodic section of the tether would be operating in the space-charge-limited regime for the most efficient ribbon tethers, thus relaxing the need to obtain work functions down to the $0.6-\mathrm{eV}$ level over the entire cathodic section.

\section{REFERENCES}

[1] Y. Toda, H. Yanagi, E. Ikenaga, J. J. Kim, M. Kobata, S. Ueda, T. Kamiya, M. Hirano, K. Kobayashi, and H. Hosono, "Work function of a room-temperature, stable electride $\left[\mathrm{Ca}_{24} \mathrm{Al}_{28} \mathrm{O}_{64}\right]^{4+}\left(\mathrm{e}^{-}\right)_{4}, "$ Adv. Mater, vol. 19, no. 21, pp. 3564-3569, Nov. 2007.

[2] D. Warner, R. Branam, and W. Hargus, "Ignition and plume characteristics of low-current cerium and lanthanum hexaboride hollow cathodes," $J$. Prop. Power, vol. 26, no. 1, pp. 130-134, 2010.

[3] S. W. Kim, Y. Toda, K. Hayashi, M. Hirano, and H. Hosono, "Synthesis of a room temperature stable $12 \mathrm{CaO} \cdot 7 \mathrm{Al}_{2} \mathrm{O}_{3}$ electride from the melt and its application as an electron field emitter," Chem. Mater., vol. 18, no. 7 , pp. 1938-1944, Apr. 2006.

[4] J. E. Medvedeva, E. N. Teasley, and M. D. Hoffman, "Electronic band structure and carrier effective mass in calcium aluminates," Phys. Rev. B, vol. 76 , no. 15 , pp. $155107-1-155107-6$, Oct. 2007.

[5] Y. Toda, S. Matsuishi, K. Hayashi, K. Ueda, T. Kamiya, M. Hirano, and $\mathrm{H}$. Hosono, "Field emission of electron anions clathrated in subnanometer-sized cages in $\left[\mathrm{Ca}_{24} \mathrm{Al}_{28} \mathrm{O}_{64}\right]^{4+}\left(\mathrm{e}^{-}\right), "$ Adv. Mater. vol. 16, no. 8, pp. 685-689, Apr. 2004.

[6] S. Matsuishi, Y. Toda, M. Miyakawa, K. Hayashi, T. Kamiya, M. Hirano, I. Tanaka, and $\mathrm{H}$. Hosono, "High-density electron anions in a nanoporous single crystal: $\left[\mathrm{Ca}_{24} \mathrm{Al}_{28} \mathrm{O}_{64}\right]^{4+}\left(4 \mathrm{e}^{-}\right), "$ Science, vol. 301 , no. 5633 , pp. 626-629, Aug. 2003.

[7] S. W. Kim, S. Matsuishi, M. Miyakawa, K. Hayashi, M. Hirano, and $\mathrm{H}$. Hosono, "Fabrication of room temperature-stable $12 \mathrm{CaO} \cdot 7 \mathrm{Al}_{2} \mathrm{O}_{3}$ electride: A review," J. Material Science, vol. 18, no. S1, pp. S5-S14, Oct. 2007.

[8] J. R. Sanmartin, M. Charro, J. Pelaez, I. Tinao, S. Elaskar, A. Hilgers, and M. Martinez-Sanchez, "Floating bare tether as upper atmosphere probe," J. Geophys. Res., vol. 111, no. A11, pp. A11310-1-A11310-11, Nov. 2006

[9] J. R. Sanmartin and R. D. Estes, "The orbital-motion-limited regime of cylindrical Langmuir probes," Phys. Plasmas, vol. 6, no. 1, pp. 395-405, Jan. 1999.

[10] I. Langmuir and K. B. Blodgett, "Currents limited by space charge between coaxial cylinders," Phys. Rev., vol. 22, no. 4, pp. 347-356, Oct. 1923. 